\title{
Antisense of survivin inhibits cervical cancer growth in mice
}

Hui Xu, Tingting Liang, Yongxia Yang, Yanlei Dong, Lin Zhu

Department of Gynecology, the Second Hospital, Shandong University, Jinan, China

Submitted: 22 July 2016

Accepted: 22 November 2016

Arch Med Sci 2019; 15 (5): 1345-1351

DOI: https://doi.org/10.5114/aoms.2017.71069

Copyright $\odot 2017$ Termedia \& Banach

\section{Abstract}

Introduction: It is proposed that survivin plays a critical role in the pathogenesis of cancer. Immune regulatory cells are associated with the growth of cancer in the body. Antisenses for the key molecules can suppress tumor growth. This study tests the hypothesis that the antisense of survivin can inhibit cervical cancer.

Results: The results showed that human cervical cancer cells expressed high levels of survivin. The levels of survivin in cervical cancer positively correlated with the frequency of interleukin (IL)-10-producing B cells (B10 cells) in the cancer tissue. Survivin increased the expression of IL-10 in B cells. Exposure to survivin antisense efficiently decreased IL-10 expression in B cells. Administration of antisense of survivin inhibited cervical cancer growth and reduced the frequency of $B 10$ cells in tumor-bearing mice.

Conclusions: The results suggest that the survivin antisense has the potential to be used in the treatment of cervical cancer.

Key words: cervical cancer, surviving, interleukin-10, B lymphocyte.

\section{Introduction}

Cervical cancer is one of the cancers in the female population [1]. The prevalence of cervical cancer has been rising in recent years [2] with unknown pathogenesis [3]. Because of the anatomical features, early diagnosis of female genital cancer is not common; most patients are at advanced stages at the time of diagnosis, although cervical cancer is easier to diagnose than ovarian cancer and endometrial cancer [4]. The treatment of cervical cancer is not satisfactory currently [5]. Therefore, it is important to invent novel therapies for cervical cancer.

Survivin is a protein encoded by the BIRC5 gene in humans; it is also called baculoviral inhibitor of apoptosis repeat-containing 5 or BIRC5 [6]. Survivin belongs to the apoptosis inhibitor family; it specifically inhibits caspase activation, which results in negative regulation of apoptosis [7]. Published data indicate that the breakdown of survivin expression can inhibit cancer growth [8]. It is also reported that most cancer cells express survivin while most terminal body cells do not [9]. This fact implies that survivin is an optimal potential target of cancer treatment.

Upon activation, regulatory B cells produce interleukin (IL)-10 to suppress other effector immune cell activities, such as to inhibit cytotoxic $\mathrm{CD}^{+} \mathrm{T}$ cell functions; thus, they are also called B10 cells [10]. Similar to the regulatory $\mathrm{T}$ cells, B10 cells are beneficial to the tumor escaping

\author{
Corresponding author: \\ Dr. Lin Zhu \\ Department of Gynecology \\ The Second Hospital \\ Shandong University \\ 247 Beiyuan St \\ Jinan 250033, China \\ E-mail: linrzhu@outlook.com
}


from immune surveillance by interfering with the anti-tumor immunity [11]. On the other hand, B10 cells also inhibit apoptosis, which may contribute to cancer development [12]. Published data indicate that survivin and IL-10 increase in cancer-bearing mice [13]. Thus, we hypothesize that survivin may up-regulate the expression of IL-10 in B cells. In this study, we found that the levels of survivin were higher in cervical cancer than normal tissue. A positive correlation was identified between survivin and IL-10 mRNA in intratumoral $B$ cells. Administration of survivin antisense efficiently inhibited cervical cancer growth and decreased the frequency of B10 cells in the cervical cancer tissue in a mouse model.

\section{Material and methods}

\section{Patients}

Ten patients with cervical cancer were recruited to this study. The diagnosis and treatment of cervical cancer were performed by doctors in our department following routine procedures. Patients treated with immune suppressive agents or radiotherapy or with autoimmune diseases were excluded from the study. The demographic data of the patients are presented in Table I. The experimental procedures were approved by the Human Ethics Committee at Shandong University. Informed written consent was obtained from each patient.

\section{Preparation of single cells with surgically removed cervical cancer}

Cervical cancer tissue was collected from the operation room immediately after removal from the patients. The tissue was cut into small pieces (about $2 \times 2 \times 2 \mathrm{~mm}$ ) and incubated with $0.5 \mathrm{mg} / \mathrm{ml}$ collagen IV (Sigma Aldrich) for $2 \mathrm{~h}$ at $37^{\circ} \mathrm{C}$ with mild agitation. The cells were harvested by filtering through a cell strainer $(40 \mu \mathrm{m})$ and centrifugation.

Table I. Demographic data

\begin{tabular}{|lc|}
\hline Characteristics & Value \\
\hline Age [years] & $54.8 \pm 15.6$ \\
\hline Disease stage: & $4(40 \%)$ \\
\hline $\mathrm{Ib} 2$ & $3(30 \%)$ \\
\hline $\mathrm{Ila}$ & $3(30 \%)$ \\
\hline Ilb & $4.5(2.8-5.5)$ \\
\hline Lymph node metastasis: & $1(10 \%)$ \\
\hline Para-aortic nodes & $1(10 \%)$ \\
\hline Parametrial invasion & $2(20 \%)$ \\
\hline Bladder/rectal invasion & $1(10 \%)$ \\
\hline
\end{tabular}

\section{Isolation of B cells}

By magnetic cell sorting (MACS) with commercial reagent kits (Miltenyi Biotech) following the manufacturer's instructions, CD19+ $\mathrm{B}$ cells were isolated from the single cell preparation of cervical cancer as described above, or from the peripheral blood mononuclear cells collected from healthy subjects. The B cell purity was $99 \%$ as checked by flow cytometry.

\section{Cell culture}

B cells were cultured in RPMI1640 medium supplemented with $10 \%$ fetal bovine serum, $100 \mathrm{U} / \mathrm{ml}$ penicillin, $0.5 \mathrm{mg} / \mathrm{ml}$ streptomycin, $2 \mathrm{mM}$ L-glutamine and $20 \mathrm{ng} / \mathrm{ml}$ anti-CD40 mAb. The medium was changed in 2-3 days. The viability of the cells was greater than $99 \%$ as checked by the trypan blue exclusion assay before being used for further experiments.

\section{Real time quantitative RT-PCR (RT-qPCR)}

Total RNAs were extracted from B cells or cervical cancer tissue with TRYzol reagents (Invitrogen). The cDNA was synthesized with the RNA samples and a reverse transcription kit (Invitrogen). The samples were then amplified on a real-time PCR device (CFX96 Touch; Bio Rad) with the SYBR Green Master Mix (Invitrogen). The primers used in the present study include tgacccatggactgaacaca and acagtcacagagagtccagc (survivin), and gttctttggggagccaacag and gctccctggtttctcttcct (IL-10). The results were calculated with the $2^{-\Delta \Delta \mathrm{ct}}$ method and are presented as fold change compared to the control group.

\section{Western blotting}

The total proteins were extracted from the cervical cancer tissue. The proteins were fractioned with SDS-PAGE (sodium dodecyl sulfate polyacrylamide gel electrophoresis) and transferred onto a PVDF membrane. After blocking with 5\% skim milk for $30 \mathrm{~min}$ at room temperature, the membrane was incubated with anti-survivin mAb (1 : 200; Santa Cruz Biotech) overnight. The membrane was then washed with TBST (Tris-buffered saline Tween 20) 3 times with shaking, followed by incubating with peroxidase-labeled second antibodies (Santa Cruz Biotech) for $1 \mathrm{~h}$ at room temperature and washed again with TBST 3 times. The immune complex on the membrane was developed with ECL (enhanced chemiluminescence; GE Healthcare). The results were photographed with an image system (UVI, Cambridge, UK).

\section{Assessment of the capacity of survivin for regulation of IL-10 expression in B cells}

$\mathrm{CD} 19^{+} \mathrm{B}$ cells were isolated from the peripheral blood samples collected from healthy controls. 
B cells were cultured $\left(10^{6}\right.$ cells $/ \mathrm{ml}$; each sample was tested in triplicate) in the presence of recombinant survivin (R\&D Systems) at gradient concentrations for $48 \mathrm{~h}$. The B cells were then harvested to be analyzed by RT-qPCR to determine the IL-10 mRNA levels.

\section{Preparation of antisense of survivin- carrying liposomes}

The antisense of survivin was designed, synthesized and constructed into liposomes by Enke Biotech (Shenzhen, China). The sequences of the antisense tested by Enke Biotech are listed in Table II, from which the No. 2 sequence was used in the study.

\section{Preparation of cervical cancer cell line}

U14 cells, a cell line of mouse cervical cancer, were obtained from the Cell Bank at Union Medical University (Beijing, China). The cells were cultured in DMEM (Dulbecco's Modified Eagle Medium) supplemented with $10 \%$ fetal bovine serum, $100 \mathrm{U} / \mathrm{ml}$ penicillin, $0.5 \mathrm{mg} / \mathrm{ml}$ streptomycin and $2 \mathrm{mM}$ L-glutamine. The medium was changed every day. The cell viability was greater than $99 \%$ as checked by the trypan blue exclusion assay before use in further experiments.

\section{Assessment of the effects of antisense of survivin on suppression of survivin expression in cervical cancer cells}

U14 cells $\left(10^{6}\right.$ cells $\left./ \mathrm{ml}\right)$ were treated with the antisense of survivin-carrying liposomes (provided by Enke Biotech, Shenzhen, China) in the culture at $1 \mu \mathrm{g} / \mathrm{ml}$ (suggested by the manufacturer) for $48 \mathrm{~h}$. The cells were then harvested and analyzed by RT-qPCR to determine the levels of survivin in the cells. Each sample was tested in triplicate. Each experiment was repeated 3 times.

\section{Mice}

Female C57B6 mice were purchased from the Shanghai Xinmao Experimental Animal Center (Shanghai, China). The mice were maintained in a pathogen-free environment and allowed to access food and water freely. The experimental procedures were approved by the Animal Care Committee at Shandong University (approval number: SDU20150023).

Preparation of cervical cancer-bearing mice and treatment with antisense of survivin

The mice were subcutaneously injected with U14 cells ( $10^{6}$ cells/mouse) at the back skin. The mice were grouped $(n=10)$ into the saline group (treated with saline), antisense group (treated with antisense of survivin-liposomes, $0.5 \mathrm{mg} /$ mouse, daily intraperitoneal injection from day 7 to day 12) and the control group (treated with empty liposomes). The tumor size $(4 / 3 \times 3.14 \times$ $\left.\left(a \times b \times c^{*}\right)\right)$ in each mouse was recorded daily. ( ${ }^{*} a, b$ and $c$ are the half size of height, width and length of the tumor).

\section{Assessment of the frequency of B10 cells in experimental cervical cancer tissue}

After sacrifice, the tumor mass was removed from each mouse. Single cells were prepared with the tumor tissue as described above. The cells were stained with FITC-labeled anti-CD19 antibody (or isotype IgG) for $30 \mathrm{~min}$ at $4^{\circ} \mathrm{C}$. After washing with phosphate-buffered saline (PBS), the cells were fixed with $1 \%$ paraformaldehyde for $1 \mathrm{~h}$ at room temperature, followed by incubation with $0.5 \%$ saponin for $30 \mathrm{~min}$. The cells were then incubated with APC-labeled anti-IL-10 antibody (or isotype IgG) for $30 \mathrm{~min}$ at $4^{\circ} \mathrm{C}$. After washing with PBS, the cells were analyzed with a flow cytometer (FACSCanto II, BD Bioscience). The data were analyzed with Flowjo (TreeStar) with the isotype IgG staining data as a gating reference.

\section{Statistical analysis}

Data are presented as mean \pm SD. The difference between two groups was determined by Student's $t$ test or ANOVA if more than two groups. The correlation between two groups was assessed by Microsoft Excel. $P<0.05$ was set as the criterion for significance.

\section{Results}

\section{High levels of survivin are detected in cervical cancer}

To investigate the role of survivin in the pathogenesis of cervical cancer, we collected 10 samples from surgically removed cervical cancer in the operation room. The samples were analyzed by RT-qPCR and Western blotting. The results showed that the levels of survivin were significantly higher in cancer tissue than those in normal tissue (Figure 1). The results indicate that survivin may be associated with the development of cervical cancer.

Table II. Sequences of survivin antisense

\begin{tabular}{|lc|}
\hline Sequence number & \multicolumn{1}{c|}{ Sequences } \\
\hline 1 & TGTTAAACAGCAGGTTCAGCCTTTA \\
\hline 2 & ACAGCAGGTTCAGCCTTTATGACAA \\
\hline 3 & CATGAGTTACGAGAGCACAGCTTTG \\
\hline 4 & CAGAGTGTAGCTGCACACCTGACAA \\
\hline 5 & CAGTGAGAATGAACCTGATGTCGTT \\
\hline
\end{tabular}




\section{IL-10 levels in intra-cervical cancer}

B cells are correlated with survivin levels in cervical cancer

To elucidate the relation between survivin and the expression of IL-10 in B cells, we measured the levels of IL-10 mRNA in B cells isolated from cervical cancer tissue. The results showed that the levels of IL-10 mRNA in the cancer tissue-isolated $B$ cells were higher than those in peripheral $B$ cells (Figure 2 A). A correlation assay was performed with the data of IL-10 and survivin. The results showed a positive correlation between the IL-10 mRNA levels and the survivin levels in the cervical tissue (Figure 2 B). The results suggest that survivin may play a role in up-regulating IL-10 expression in B cells.

\section{Survivin increases IL-10 expression in B cells}

The data reported above suggest that survivin may up-regulate the expression of IL-10 in B cells. To test this, we isolated $B$ cells from healthy per- sons. The B cells were cultured in the presence of survivin for $48 \mathrm{~h}$. As analyzed by RT-qPCR and Western blotting, the presence of survivin in the culture markedly increased the expression of IL-10 in B cells at both mRNA and protein levels in a survivin dose-dependent manner (Figure 3). The results demonstrate that survivin does increase the expression of IL-10 in B cells.

\section{Antisense of survivin inhibits cervical cancer growth in mice}

The data presented in Figures 1-3 suggest that survivin may contribute to cervical cancer growth. To test this, we designed antisense of survivin. As tested by the cell culture study, the designed antisense sequences showed suppressor effects on survivin expression in U14 cells (a mouse cervical cancer cell line) (Figure 4). We then developed a mouse model of cervical cancer with U14 cell transplantation. The cancer-bearing mice were treated with the survivin antisense-carrying liposomes daily for 9 days. From the tumor size re-
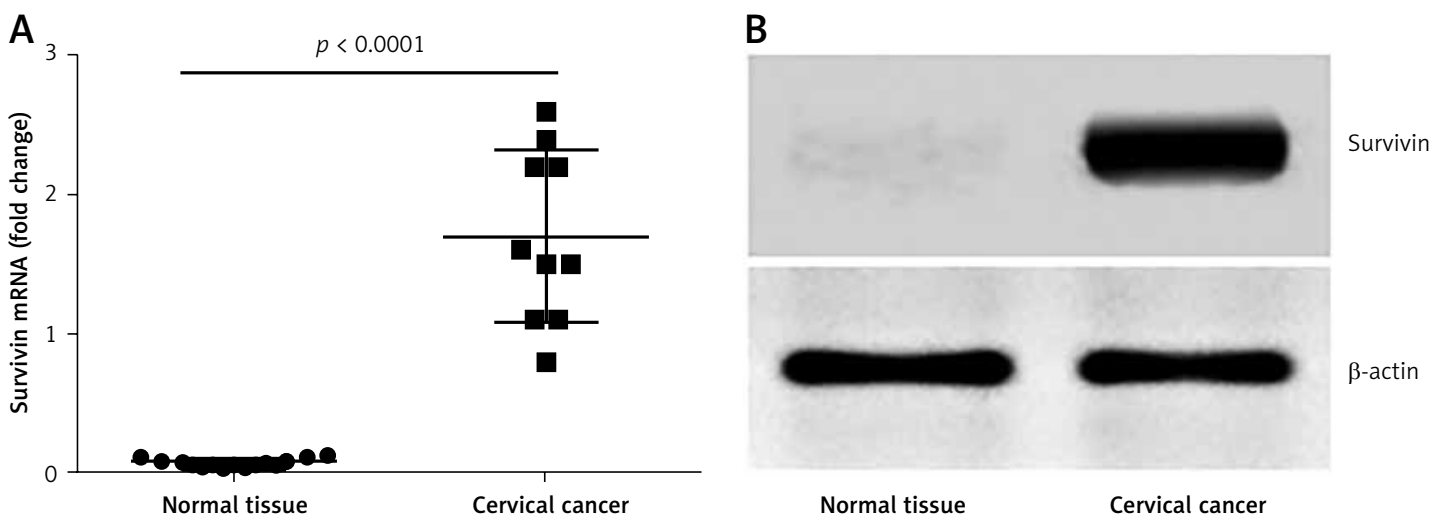

Figure 1. Survivin levels in cervical cancer tissue. A - The scatter dot plots show the survivin mRNA levels in cervical cancer tissue and the marginal normal tissue of patients with cervical cancer $(n=10)$. B - The representative immune blots show the protein levels of survivin in cervical cancer tissue and normal tissue from 10 patients with cervical cancer
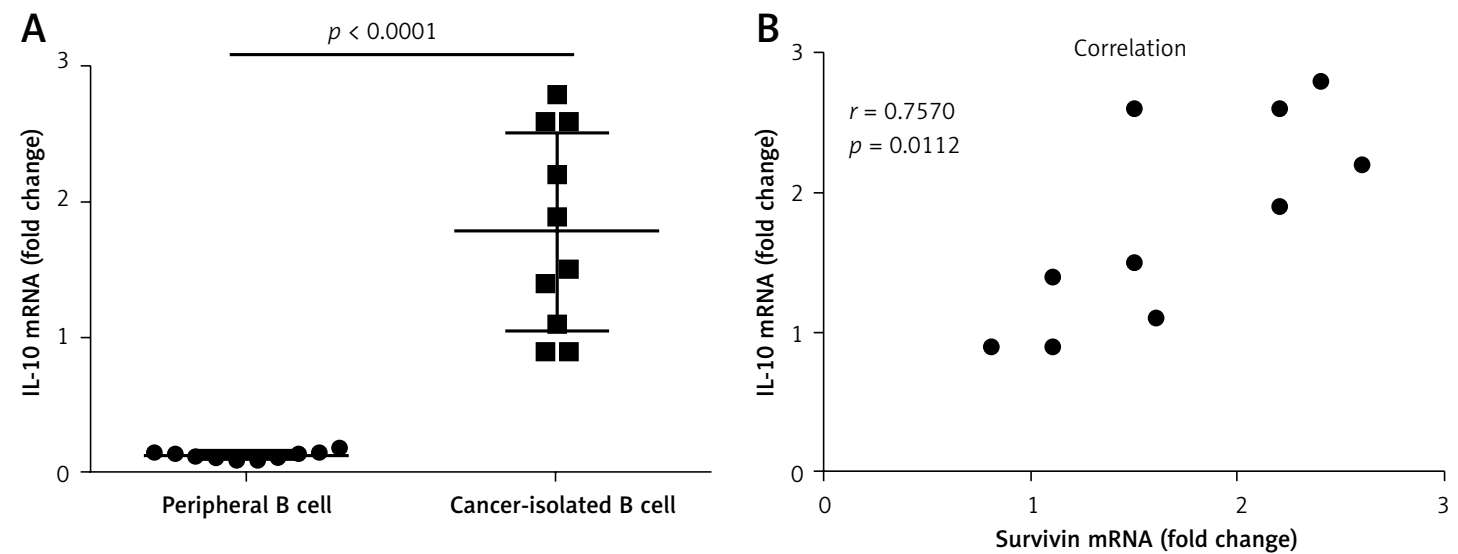

Figure 2. Assessment of the correlation between survivin in cervical cancer and IL-10 in intratumoral B cells. A - The scatter dot plots show the IL-10 mRNA levels in the intratumoral B cells and the peripheral B cells. B - The scatter dot plots show the correlation between survivin in cervical cancer tissue and the IL-10 mRNA levels in the intratumoral B cells 
A

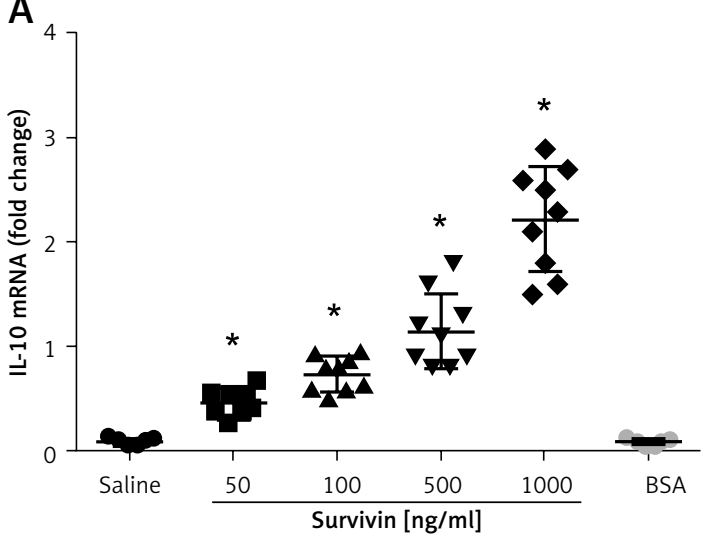

B

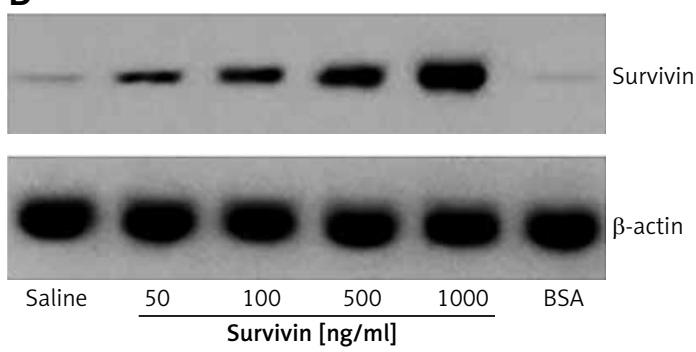

Figure 3. Survivin increases the expression of IL-10 in B cells. A - The scatter dot plots show the IL-10 mRNA levels in B cells after culturing in the presence of survivin (as denoted on the X axis) for $48 \mathrm{~h}$. Each dot represents an individual datum. ${ }^{*} p<0.0001$, compared with the saline group. BSA: bovine serum albumin; used as a control agent $(1000 \mathrm{ng} / \mathrm{ml})$. B - The immune blots indicate the protein levels of survivin. The data were from 3 independent experiments; three samples were tested in triplicate in each experiment

cords, we found that administration of survivin antisense efficiently inhibited the cervical cancer growth (Figure 5). We also assessed the frequency of B10 cells in the tumor. The results showed that administration of survivin antisense significantly decreased the frequency of B10 cells in the tumor tissue (Figure 6).

\section{Discussion}

The therapeutic efficacy of cervical cancer is unsatisfactory currently $[14,15]$. The optimal treatment time and radiation dose are critical to the therapeutic efficacy and are significantly associated with survival improvement [14]. Thus, it is important to invent novel therapeutic remedies for cervical cancer. The present study aims to assess the effects of antisense of survivin on cervical cancer. From the cervical cancer tissue which we

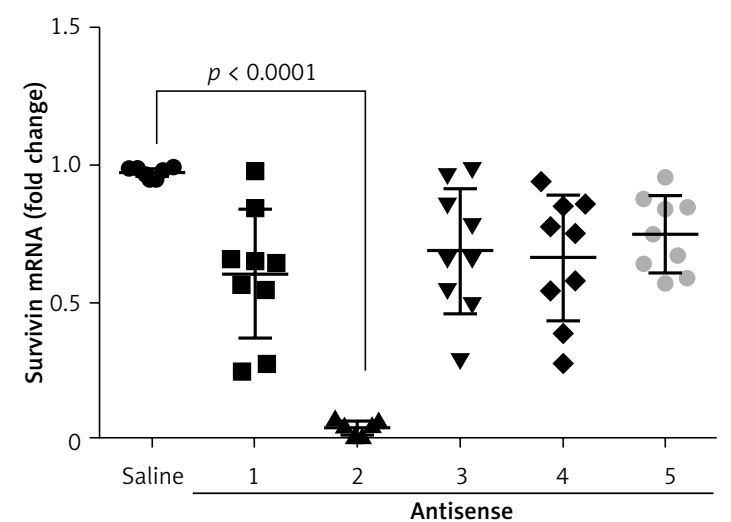

Figure 4. Effects of antisense on survivin expression in cervical cancer cells. The scatter dot plots show the survivin mRNA levels in U14 cells after treatment with saline or antisense of survivin-carrying liposomes. The data were from 3 independent experiments; three samples were tested in triplicate in each experiment collected from patients with cervical cancer tissue we found high levels of survivin in cervical cancer. We also found high frequency of B10 cells in cervical cancer. Because a positive correlation was identified between the frequency of B10 cells and the survivin levels in cervical cancer, we treated $B$ cells collected from healthy persons with recombinant survivin in the culture. The results showed that exogenous survivin markedly increased the expression of IL-10 in B cells. By treating tumor-bearing mice with antisense of survivin, we found that the antisense of survivin efficiently inhibited cervical cancer growth.

If the cervical cancer is diagnosed at the early stage without metastasis, treatment with surgery to radically remove the cancer can achieve good results [4]. However, if the cervical cancer is found at the advanced stage, especially those with re-

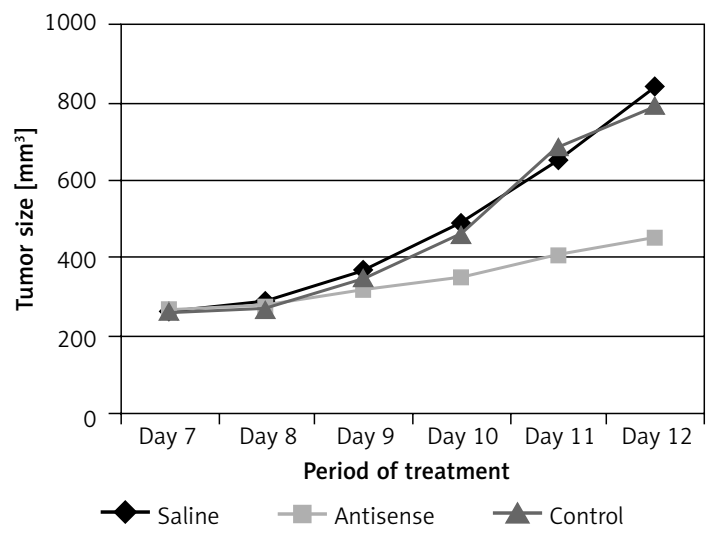

Figure 5. Records of tumor size. The curves indicate the tumor size in mice during the period of treatment as denoted in the figure. Each group consists of 10 mice 
A

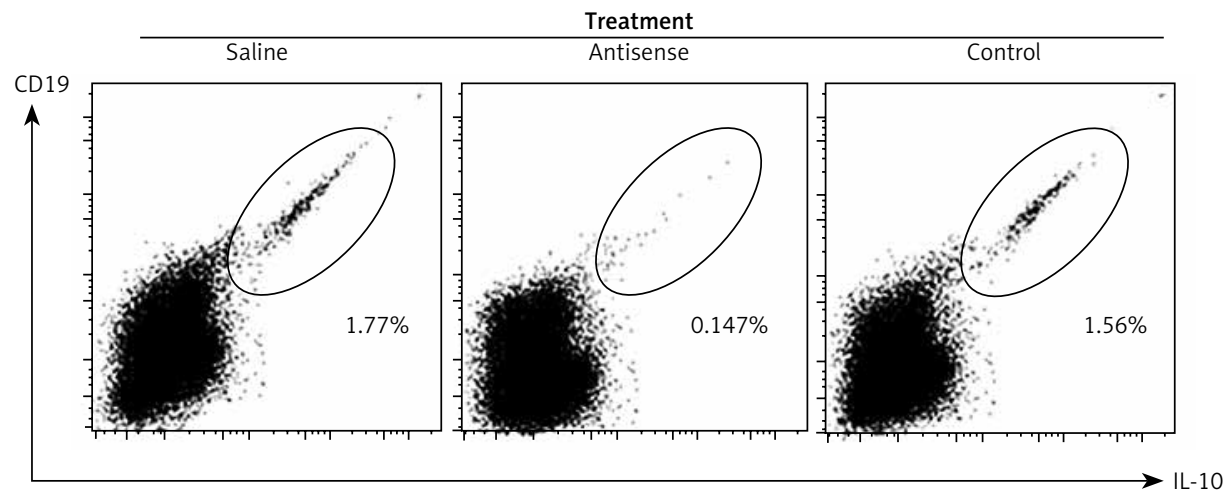

B

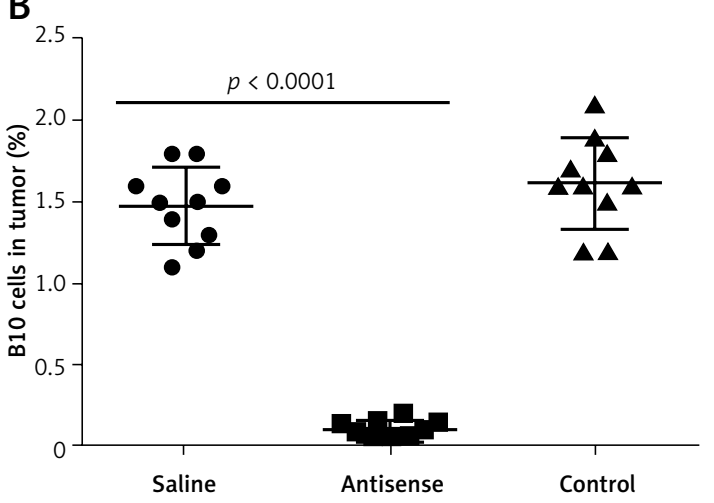

Figure 6. B10 cells in experimental cervical cancer. A - The flow cytometry dot plots show the frequency of B10 cells in the cervical cancer tissue. B - The scatter dot plots show the individual data of B10 cells in the cervical cancer tissue of each tumor-bearing mouse. Each group consists of 10 mice

mote metastasis, treatment with surgery alone may be unsatisfactory [4]; some adjuvant remedies, such as radiotherapy, chemotherapy and immunotherapy, may be employed before and/ or after surgery [16]. The present data show that gene therapy inhibiting survivin may be an additional remedy for the treatment of cervical cancer. Although using the antisense of survivin did not completely remove the experimental cervical cancer from mice, it significantly inhibited the cancer growth in mice. Therefore, this method has the potential to be a supportive method in the treatment of cervical cancer.

In this study, we identified a positive correlation between survivin and B10 cells in cervical cancer tissue collected from patients with cervical cancer. B10 cells are a fraction of immune regulatory cells. Cumulative reports indicate that immune regulatory cells, including regulatory $T$ cells and regulatory $B$ cells, play a critical role in the pathogenesis of cancer $[17,18]$. The underlying mechanism is that the immune regulatory cells are capable of suppressing the antitumor immunity, such as inhibiting CD8 ${ }^{+}$cytotoxic cells $[19,20]$.

The present data show that the $B$ cells isolated from cervical cancer tissue had high levels of IL-10 expression, which was correlated with the levels of survivin in the same cancer tissue. We understood that this phenomenon could be a coincidence or a causal relationship between survivin and IL-10 expression in B cells. With an in vitro cell culture study, we were able to demonstrate

that survivin was capable of up-regulating the IL-10 expression in B cells. The results suggest that cervical cancer cells produce survivin; the latter increases IL-10 expression in the intratumoral $B$ cells to confer the B cells with immune suppressor function, which compromises the antitumor immunity in the local tissue. Thus, we designed an antisense of survivin carried by liposomes. The administration of the antisense-carrying liposome efficiently inhibited the experimental cervical cancer growth. Although the antisense could not radically remove the tumor, the tumor mass was significantly smaller than the control tumor mass.

In conclusion, the present study revealed that cervical cancer expressed high levels of survivin, which was correlated with the expression of IL-10 in the intratumoral B cells. Administration of antisense of survivin efficiently inhibited the experimental cervical cancer growth and decreased the frequency of B10 cells in the cervical cancer. The data suggest that the antisense of survivin may be a potential adjuvant therapeutic remedy for cervical cancer.

\section{Acknowledgments}

This study was supported by a grant from the Shandon Provincial Science \& Technology Advance Foundation (2011GGE27110).

\section{Conflict of interest}

The authors declare no conflict of interest. 


\section{References}

1. Tsikouras P, Zervoudis S, Manav B, et al. Cervical cancer: screening, diagnosis and staging. J BUON 2016; 21 : 320-5.

2. Lee H, Kang Y, Ju W. Cervical cancer screening in developing countries: using visual inspection methods. Clin J Oncol Nurs 2016; 20: 79-83.

3. Chanthavilay P, Mayxay M, Phongsavan K, et al. Accuracy of combined visual inspection with acetic acid and cervical cytology testing as a primary screening tool for cervical cancer: a systematic review and meta-analysis. Asian Pac J Cancer Prev 2015; 16: 5889-97.

4. Li H, Wu X. Advances in diagnosis and treatment of metastatic cervical cancer. J Gynecol Oncol 2016; 27: e43.

5. Zhu H, Luo H, Shen Z, Hu X, Sun L, Zhu X. Transforming growth factor-beta1 in carcinogenesis, progression, and therapy in cervical cancer. Tumour Biol 2016; 37: 7075-83.

6. Garg H, Suri P, Gupta JC, Talwar GP, Dubey S. Survivin: a unique target for tumor therapy. Cancer Cell Int 2016; 16: 49.

7. Athanasoula K, Gogas H, Polonifi K, Vaiopoulos AG, Polyzos A, Mantzourani M. Survivin beyond physiology: orchestration of multistep carcinogenesis and therapeutic potentials. Cancer Lett 2014; 347: 175-82.

8. Chen X, Duan N, Zhang C, Zhang W. Survivin and tumorigenesis: molecular mechanisms and therapeutic strategies. J Cancer 2016; 7: 314-23.

9. Ryan BM, O'Donovan N, Duffy MJ. Survivin: a new target for anti-cancer therapy. Cancer Treat Rev 2009; 35: 553-62.

10. Dai K, Huang L, Chen J, Yang L, Gong Z. Amphiregulin promotes the immunosuppressive activity of intrahepatic $C D 4+$ regulatory $T$ cells to impair $C D 8+T$ cell immunity against hepatitis $B$ virus infection. Immunology 2015; 144: 506-17.

11. Zhang Y, Morgan R, Podack ER, Rosenblatt J. B cell regulation of anti-tumor immune response. Immunol Res 2013; 57: 115-24.

12. Gray M, Gray D. Regulatory B cells mediate tolerance to apoptotic self in health: implications for disease. Int Immunol 2015; 27: 505-11.

13. Abdin AA, Soliman NA, Saied EM. Effect of propranolol on IL-10, visfatin, Hsp70, iNOS, TLR2, and survivin in amelioration of tumor progression and survival in solid Ehrlich carcinoma-bearing mice. Pharmacol Rep 2014; 66: 1114-21.

14. Manders DB, Moron A, McIntire D, et al. Locally advanced cervical cancer: outcomes with variable adherence to treatment. Am J Clin Oncol 2016 in press.

15. Todo Y, Watari H. Concurrent chemoradiotherapy for cervical cancer: background including evidence-based data, pitfalls of the data, limitation of treatment in certain groups. Chin J Cancer Res 2016; 28: 221-7.

16. Bourgioti C, Chatoupis K, Moulopoulos LA. Current imaging strategies for the evaluation of uterine cervical cancer. World J Radiol 2016; 8: 342-54.

17. Zhang Y, Eliav Y, Shin SU, et al. B lymphocyte inhibition of anti-tumor response depends on expansion of Treg but is independent of B-cell IL-10 secretion. Cancer Immunol Immunother 2013; 62: 87-99.

18. Xie Y, Zhang X, Zhao T, Li W, Xiang J. Natural CD8(+)25(+) regulatory $T$ cell-secreted exosomes capable of suppressing cytotoxic $T$ lymphocyte-mediated immunity against B16 melanoma. Biochem Biophys Res Commun 2013; 438: 152-5.

19. Siewe B, Wallace J, Rygielski S, et al. Regulatory B cells inhibit cytotoxic T lymphocyte (CTL) activity and elimi- nation of infected CD4 T cells after in vitro reactivation of HIV latent reservoirs. PLoS One 2014; 9: e92934.

20. Siewe B, Stapleton JT, Martinson J, et al. Regulatory B cell frequency correlates with markers of HIV disease progression and attenuates anti-HIV CD8(+) T cell function in vitro. J Leukoc Biol 2013; 93: 811-8. 